\title{
Few-cycle spatiotemporal optical solitons in waveguide arrays
}

\author{
Hervé Leblond, ${ }^{1}$ David Kremer, ${ }^{2}$ and Dumitru Mihalache ${ }^{3}$ \\ ${ }^{1}$ Laboratoire de Photonique d'Angers, EA 4464, LUNAM Université, Université d'Angers, 2 Boulevard Lavoisier, 49000 Angers, France \\ ${ }^{2}$ Laboratoire MOLTECH-Anjou, CNRS UMR 6200, LUNAM Université, Université d'Angers, \\ 2 Boulevard Lavoisier, 49045 Angers Cedex, France \\ ${ }^{3}$ Horia Hulubei National Institute for Physics and Nuclear Engineering, 30 Reactorului, Magurele-Bucharest RO-077125, Romania
}

(Received 21 November 2016; published 25 April 2017)

\begin{abstract}
We consider the propagation of Gaussian spatiotemporal wave packets in arrays of parallel optical waveguides, assuming linear and nondispersive coupling between the adjacent guides. The numerical analysis is based on a discrete version of the modified Korteweg-de Vries equation that adequately describes the propagation of ultrashort (few-cycle) spatiotemporal solitons in waveguide arrays. Two kinds of such discrete-continuous localized wave forms, which are discrete solitons in the transverse direction, and few-cycle solitons in the longitudinal one, are put forward, namely breathing solitons and single-humped ones. The conditions of formation of these localized spatiotemporal structures, their time duration and spatial width, as well as their energies, are also investigated.
\end{abstract}

DOI: 10.1103/PhysRevA.95.043839

\section{INTRODUCTION}

The key features of nonlinear discrete optical systems have been extensively explored during the past several years, and arrays of evanescently coupled nonlinear waveguides have provided a fertile ground for the study of the interplay between discreteness and nonlinearity. In these specific optical settings the dispersion and diffraction properties of propagating light can be properly controlled and engineered, and many kinds of discrete optical solitons have been studied both theoretically and experimentally; see the extensive reviews [1-3]. Discrete nonlinear dynamical systems were investigated both theoretically and experimentally not only in the optics context, but also in other physical systems involving nonlinear lattices [4,5]. In these physical settings, the interplay of nonlinearity with lattice discreteness leads to unique phenomena that are quite distinct from those occurring in the corresponding continuous nonlinear dynamical systems [4]. It is also worth mentioning the intense work done during the past several years in the study of diverse mathematical models of such discrete solitons in a series of relevant physical settings [6,7]. The many mathematical models deal with the study of the nonintegrable discrete nonlinear Schrödinger (NLS) equation and the integrable Ablowitz-Ladik equation [8,9] and the investigation of other more general evolution equations that interpolate between these two generic nonlinear differentialdifference equations; see $[4,6,7]$.

In a pioneering earlier work, Christodoulides and Joseph [10] theoretically investigated the problem of discrete selffocusing in nonlinear arrays of coupled waveguides and the characteristic properties of the corresponding one-dimensional (1D) discrete solitons. One decade after the publication of the theoretical prediction [10], such 1D discrete optical solitons were experimentally observed in 1998 by Eisenberg et al. [11]. Several theoretical works investigated the formation and the robustness to propagation of both $1 \mathrm{D}$ and higher-dimensional discrete solitons in a large variety of physical settings [12-22].

The unique features of optical solitons in discrete dissipative structures have been also theoretically investigated. The existence and the instability dynamics of both 1D [23] and twodimensional (2D) [24] discrete Ginzburg-Landau solitons have been explored theoretically and numerically. Also, the motion and stability properties of such dissipative solitons, which form in multiple waveguide structures, were investigated in detail by Soto-Crespo et al. [25]. These kinds of dissipative solitons are described by the discrete complex cubic-quintic GinzburgLandau equation; see Refs. [23-25]. It is worth mentioning that, as a result of discreteness of the underlying dissipative system, the discrete dissipative optical solitons exhibit features that have no counterpart in either continuous or in conservative discrete models; see Ref. [23]. Also, in the framework of the continuous-discrete complex cubic-quintic Ginzburg-Landau model, spatiotemporal dissipative solitons that are confined inside 2D photonic lattices were also theoretically investigated [26].

Discrete vortex solitons have been investigated theoretically by Malomed and Kevrekidis [27], and subsequent works by Leblond et al. [28,29] have studied in detail the existence and stability domains of families of spatiotemporal vortex solitons in either square or hexagonal arrays of evanescently coupled waveguides.

On the experimental arena in this broad area, we mention here the series of experimental works including the observation of discrete spatial optical solitons in optically induced nonlinear photonic lattices [30-32], the observation of both 1D and 2D discrete surface solitons in waveguide arrays [33,34], the observation of three-dimensional (3D) discrete-continuous $\mathrm{X}$ waves in photonic lattices [35] and of 3D discrete-continuous spatiotemporal solitons (alias light bullets) in 2D arrays of coupled waveguides [36], and the observation of vortex light bullets that are discrete spatiotemporal solitons with embedded orbital angular momenta [37].

Several theoretical studies in the area of propagation of ultrashort light pulses in diverse physical settings have used the slowly varying envelope approximation (SVEA) and different kinds of generalizations of the generic NLS equation; see, for example, Refs. [38,39]. However, during the past years, a lot of theoretical works have explored the problem of propagation of few-cycle pulses and solitons beyond the commonly used SVEA. We only mention here the so-called unidirectional pulse propagation model [40,41], 
the Maxwell-Duffing description of ultrashort optical pulses in nonresonant media [42], and the Maxwell-Drude-Bloch model of few-cycle optical solitons [43]; for recent overviews of the main theoretical approaches in the area of nonlinear optics of ultrashort light pulses including few-cycle optical solitons, see Refs. [44-47]. The theoretical models beyond the SVEA rely on the modified Korteweg-de Vries (mKdV) [48], the short-pulse [49-51], the sine-Gordon (sG) [52], the double $\mathrm{sG}[53,54]$, and the $\mathrm{mKdV}-\mathrm{sG}$ [55-57] equations; see also Refs. [58-62]. Though most of the above mentioned generic models are purely 1D ones, studies of multidimensional ultrashort optical solitons have been performed also; for example, stable few-cycle spatiotemporal optical solitons can form in quadratically nonlinear media from few-cycle input wave packets [63]. For the sake of completeness, we mention here a few papers that overviewed the intense experimental and theoretical activity in the area of multidimensional localized structures in both optical and matter-wave media [64-70].

In a recent work [71], in the framework of a non-SVEA model that is suitable for describing the propagation of ultrashort (few-cycle) solitons, the generic equations accounting for the coupling between two adjacent optical waveguides were introduced and studied numerically, showing the possibility of soliton propagation in this setup [72]. The analysis was based on the generalized Kadomtsev-Petviashvili equation, and from this equation a set of two coupled $\mathrm{mKdV}$ equations was derived [71].

In the present work we introduce and study in detail a discrete version of the $\mathrm{mKdV}$ equation. We investigate the formation of two distinct types of solitary waves from input Gaussian spatiotemporal wave packets. We also describe the characteristic features of discrete-continuous spatiotemporal solitons, which form in coupled waveguide arrays and are localized in both space and time.

The organization of this paper is as follows. In Sec. II we present the generic model describing the propagation of few-cycle spatiotemporal optical solitons in waveguide arrays, which is based on the discrete version of coupled $\mathrm{mKdV}$ equations, and we explore in this physical setting the self-focusing effect vs the combined dispersion and diffraction effects. The detailed study of families of few-cycle discretecontinuous spatiotemporal optical solitonsn is given in Sec. III. Finally, Sec. IV concludes this paper.

\section{SELF-FOCUSING EFFECT VERSUS THE COMBINED DISPERSION AND DIFFRACTION EFFECTS IN ARRAYS OF COUPLED WAVEGUIDES}

We consider a set of $2 N+1$ parallel waveguides in a planar geometry, assuming a purely linear and non-dispersive coupling. The normalized optical electric field $u_{n}$ propagating in the $n$th waveguide satisfies the following discrete version of the $\mathrm{mKdV}$ equation [71]:

$$
\partial_{z} u_{n}=-a \partial_{t}\left(u_{n}^{3}\right)-b \partial_{t}^{3} u_{n}-c \partial_{t}\left(u_{n-1}+u_{n+1}\right),
$$

which holds for $-N \leqslant n \leqslant N$ (with the convention that $u_{-N-1}$ and $u_{N+1}$ are replaced with zero).

In (1), the dimensionless variables are defined as $z=\zeta / L$, $t=\left(\tau-\zeta / V_{0}\right) / \tau_{w}$, and $u_{n}=E_{n} / E_{0}$, where $\zeta$ and $\tau$ are the time and space variables, and $E_{n}$ is the electric field. The quantities $L, \tau_{w}$, and $E_{0}$ are reference propagation length, reference time, and reference electric field, respectively. The velocity $V_{0}=c_{0} / n_{0}$ is the ratio of the light velocity $c_{0}$ in vacuum to the linear refractive index $n_{0}$ at the low-frequency limit.

The normalized nonlinear coefficient is

$$
a=\frac{\chi^{(3)}}{2 n_{0} c_{0}} \frac{L E_{0}^{2}}{\tau_{w}},
$$

where $\chi^{(3)}$ is the third-order susceptibility at the low frequency limit. The normalized dispersion parameter is

$$
b=\frac{\left(-n^{\prime \prime}\right)}{2 c_{0}} \frac{L}{\tau_{w}^{3}},
$$

where the prime denotes the derivative with respect to $\omega$. The coupling coefficient is

$$
c=\frac{I_{2}}{1+I_{1}} \frac{L \Delta n}{c_{0} \tau_{w}},
$$

where $\Delta n$ is the refractive index shift between the core and cladding of each guide, $I_{1}=\int_{-\infty}^{\infty} f_{1} f_{2} d x$ is the overlapping integral of the normal modes $f_{1}$ and $f_{2}$ of two adjacent waveguides over the entire real axis, and $I_{2}=\int_{g_{1}} f_{1} f_{2} d x=$ $\int_{g_{2}} f_{1} f_{2} d x$ is the overlapping integral of the same field profiles, over the core of one waveguide only.

Setting

$$
\begin{gathered}
\tau_{w}^{2}=\frac{-n^{\prime \prime}}{2 \Delta n} \frac{\left(1+I_{1}\right)}{I_{2}}, \\
L=c_{0} \sqrt{\frac{-n^{\prime \prime}}{2}}\left(\frac{1+I_{1}}{I_{2} \Delta n}\right)^{3 / 2}, \\
E_{0}^{2}=\frac{I_{2}}{1+I_{1}} \frac{2 n_{0} \Delta n}{\chi^{(3)}}
\end{gathered}
$$

yields $a=b=c=1$. Hence we can restrict to this situation without loss of generality. Since the overlapping integrals $I_{1}$ and $I_{2}$ are real and positive, and waveguiding requires $\Delta n>0$, Eqs. (5)-(7) require $n^{\prime \prime}<0$ and $\chi^{(3)}>0$, which are the well-known necessary conditions for spatiotemporal soliton formation.

However, both the overlapping integrals $I_{1}$ and $I_{2}$ and $\Delta n$ can be adjusted in a wide range of values. Hence, to fix the ideas, we can set $\tau_{w}=1$ fs and assume that $I_{1}, I_{2}$, and $\Delta n$ have been adjusted in such a way that $c=1$. Then, setting

$$
L=\frac{2 c_{0} \tau_{w}^{3}}{-n^{\prime \prime}}
$$

and

$$
E_{0}^{2}=\frac{-n_{0} n^{\prime \prime}}{\chi^{(3)} \tau_{w}^{2}}
$$

reduces $a$ and $b$ to 1 . Using numerical values and a Sellmeier formula pertaining to silica glass at $\lambda=1.064 \mu \mathrm{m}, n_{0}=1.450$, $n^{\prime \prime}=-0.0053 \mathrm{fs}^{2}$, and $n_{2}=0.21 \times 10^{-19} \mathrm{~m}^{2} / \mathrm{W}$ [73] [recall that the nonlinear index $n_{2}$ is related to the third order susceptibility according to $\left.n_{2}=3 \chi^{(3)} /\left(4 n_{0}^{2} \varepsilon_{0} c_{0}\right)\right]$ then the reference propagation length is $L=113 \mu \mathrm{m}$, and the reference electric field is $E_{0}=7 \times 10^{9} \mathrm{~V} / \mathrm{m}$, corresponding to a 
reference intensity $I_{0}=\frac{1}{2} n_{0} \varepsilon_{0} c_{0} E_{0}^{2}=9500 \mathrm{GW} / \mathrm{cm}^{2}$. Using parameters pertaining to a highly nonlinear glass, such as the chalcogenide glass $\mathrm{GeSe}_{4}$, still at $\lambda=1.064 \mu \mathrm{m}$, i.e., $n=2.51, n^{\prime \prime}=-0.021 \mathrm{fs}^{2}$ [74], and $n_{2}=13 \times 10^{-18} \mathrm{~m}^{2} / \mathrm{W}$ [75], we obtain $L=29 \mu \mathrm{m}, E_{0}=4.3 \times 10^{8} \mathrm{~V} / \mathrm{m}$, and $I_{0}=$ $61 \mathrm{GW} / \mathrm{cm}^{2}$. Obviously, the material used in an experimental setup must also satisfy other requirements, especially in terms of low absorption, large bandwidth, and ability to support very high optical intensities.

It is straightforwardly proved that the system of coupled mKdV-type equations (1) conserves the quantity

$$
E=\sum_{n=-N}^{N} \int_{-\infty}^{\infty} u_{n}^{2} d t,
$$

in the sense that $\partial_{z} E=0 . E$ is proportional to the optical intensity integrated over space and time, and hence we will refer to as the pulse energy below. Equation (1) derives from the Lagrangian density

$$
\mathcal{L}=\sum_{n=-N}^{N} \mathcal{L}_{n}+\mathcal{L}_{I},
$$

where the Lagrangian density corresponding to channel $n$ is

$$
\mathcal{L}_{n}=\frac{1}{2} \partial_{t} \varphi_{n} \partial_{z} \varphi_{n}+\frac{a}{4}\left(\partial_{t} \varphi_{n}\right)^{4}-\frac{b}{2}\left(\partial_{t}^{2} \varphi_{n}\right)^{2}
$$

where

$$
u_{n}=\partial_{t} \varphi_{n},
$$

and the interaction between channels is taken into account by

$$
\mathcal{L}_{I}=c \sum_{n=-N}^{N-1} \partial_{t} \varphi_{n} \partial_{t} \varphi_{n+1} .
$$

Equation (1) also conserves the Hamiltonian $H=\int_{-\infty}^{\infty} \mathcal{H} d t$, where the Hamiltonian density is defined by

$$
\mathcal{H}=\sum_{n=-N}^{N} \mathcal{H}_{n}+\mathcal{H}_{I},
$$

with

$$
\mathcal{H}_{n}=\frac{a}{4} u_{n}^{4}+\frac{b}{2} u_{n} \partial_{t}^{2} u_{n}
$$

and

$$
\mathcal{H}_{I}=\mathcal{L}_{I}=c \sum_{n=-N}^{N-1} u_{n} u_{n+1} .
$$

We solve the system of coupled mKdV-type equations (1) using a standard fourth-order Runge-Kutta numerical scheme with respect to the evolution variable $z$ in the Fourier space. Computation of the nonlinear term involves one inverse and one direct fast Fourier transform at each substep of the numerical scheme. We use normalized values of the parameters $a=b=c=1$ and periodic boundary conditions in both $n$ and $t$ directions.
We solve Eqs. (1) with the initial spatiotemporal Gaussian wave packet

$$
u_{n}(z=0, t)=A_{0} \sin (\omega t+\varphi) \exp \left(-\frac{n^{2}}{w_{0}^{2}}-\frac{t^{2}}{\tau^{2}}\right),
$$

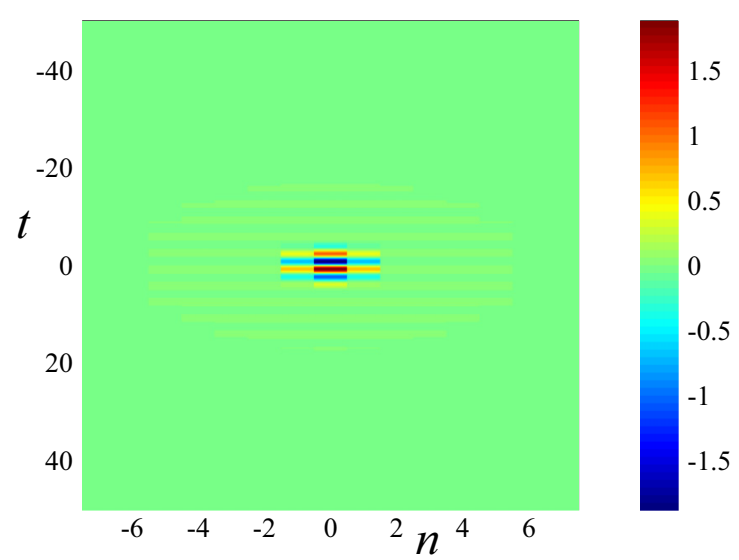

(a)

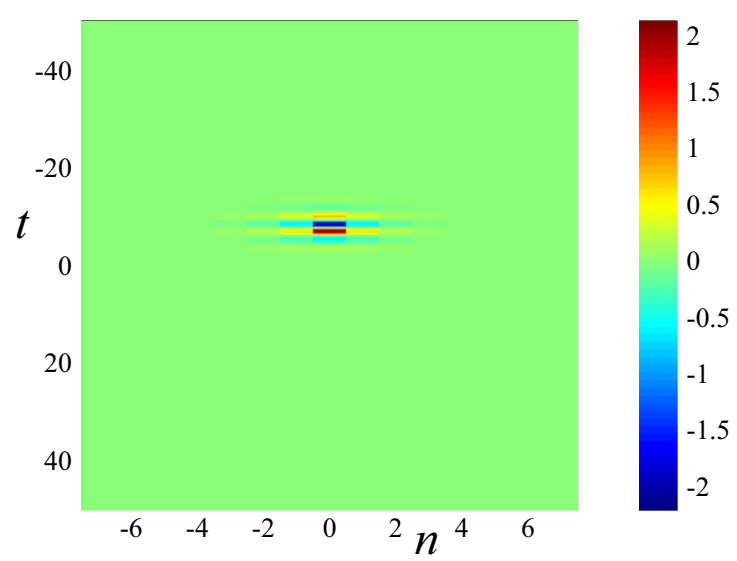

(b)

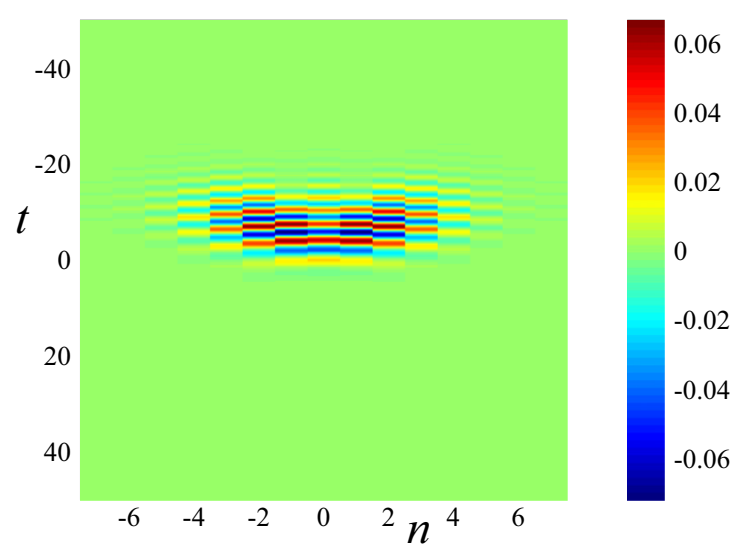

(c)

FIG. 1. Formation of solitons from a Gaussian pulse. (a) Input. (b) For a high amplitude, a soliton forms (amplitude $A_{0}=2.06$, pulse duration $f w h m=3.5$, and propagation distance $z=288$ ). (c) For a relatively low amplitude, the pulse is spread out by diffraction and dispersion (the input is the same as in (a) above, with same duration but with amplitude $A_{0}=0.2$. Propagation distance $z=0.72$ ). 


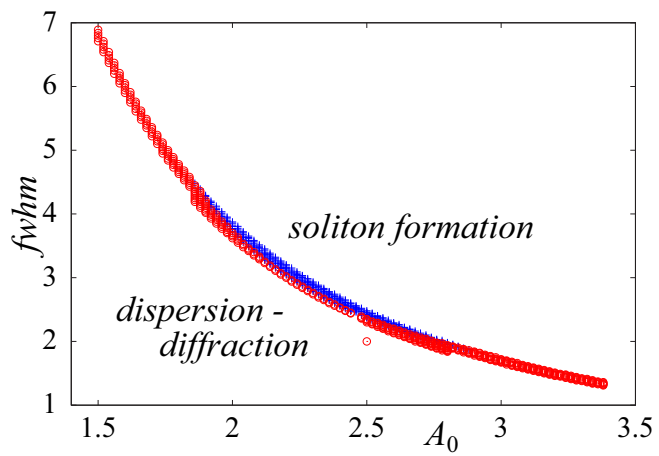

FIG. 2. Formation of solitons from a Gaussian input in the amplitude-pulse duration plane, for $w_{0}=1$. Blue crosses: soliton forms; red circles: dispersion-diffraction occurs.

so that the input light is mainly launched in the central guide, $n=0$; see Fig. 1(a). We fix the initial envelope-carrier phase as $\varphi=0$ and the initial pulse width as $w_{0}=1$. The angular frequency is $\omega=2 \pi V / \lambda$, with $\lambda=1$ and $V=0.3$, so that, if the reference time is set to $\tau_{w}=1 \mathrm{fs}$, it corresponds to a wavelength in vacuum of $1 \mu \mathrm{m}$. The initial pulse duration $\tau$ is related to its full width at half maximum $f \mathrm{whm}$ in a standard way, as $f w h m=\sqrt{2 \ln 2 \tau}$.

Then we vary the amplitude $A_{0}$ and the initial pulse duration $\tau$, and compute the evolution of the spatiotemporal wave packet. If both input amplitude $A_{0}$ and input duration $\tau$ are high enough, the light remains localized in both space and time; see Fig. 1(b). However, for relatively low input amplitudes, the combined effects of diffraction and dispersion spread out the wave packet, though it was kept a relatively high value of pulse duration $\tau$; see Fig. 1(c).

Since the soliton forms at a high input amplitude, the question arises, does this happen above some energy threshold. The limit of the domain where the solitons either form or not from Gaussian inputs is shown in Fig. 2, in the amplitude-pulse duration plane ( $A_{0}$ vs pulse duration $\left.\mathrm{fwhm}\right)$, and in Fig. 3, in the amplitude-energy plane (each symbol corresponds to a numerical calculation. In the white domain, no calculation was performed). The energy $E$ is defined by Eq. (10) above. It is seen from Fig. 3 that the energy threshold for the formation of such few-cycle discrete optical solitons is about 10; however,

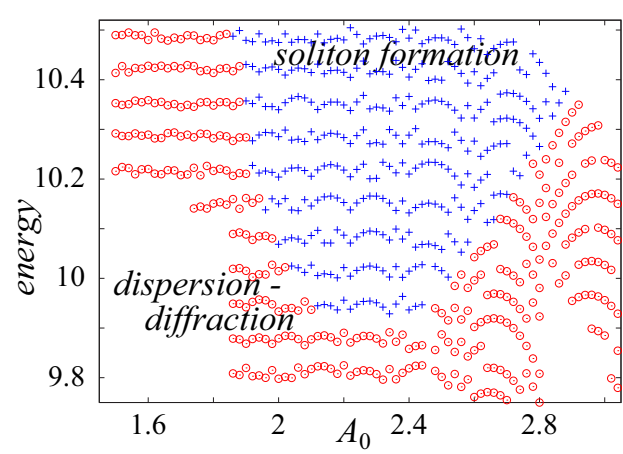

FIG. 3. Formation of solitons from a Gaussian input in the amplitude-energy plane, for $w_{0}=1$. Blue crosses: soliton forms; red circles: dispersion-diffraction occurs.

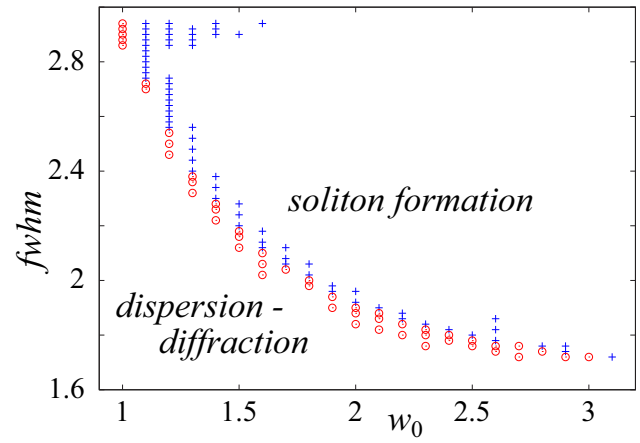

FIG. 4. Formation of solitons from a Gaussian input in the transverse width-pulse duration plane, for $A_{0}=2.22$. Blue crosses: soliton forms; red circles: dispersion-diffraction occurs.

the threshold is not independent of the input amplitude. There is some "optimum" input amplitude for which the solitons form with less energy. This optimum amplitude lies between 2.2 and 2.4; see Fig. 3.

An analogous analysis is performed against transverse width $w_{0}$, as shown on Figs. 4 and 5. The amplitude is fixed here to $A_{0}=2.22$.

It is also seen that the formation of a soliton requires a large enough input energy. However, the energy required strongly increases with $w_{0}$, and cannot be interpreted as a threshold any more, even in a rough way. This can be explained by the fact that the solitons that are formed with high values of $w_{0}$ do not have a larger width than the solitons formed with $w_{0}=1$, but are exactly the same structures. As a consequence, for large values of $w_{0}$, the shape of the input is badly matched to the final soliton, the energy is less efficiently converted into a soliton, and more total energy is required. It may even happen, for large values of $w_{0}$, that two solitons are formed instead of only one, both remaining located in the central channel $n=0$.

\section{FAMILIES OF FEW-CYCLE DISCRETE-CONTINUOUS SPATIOTEMPORAL OPTICAL SOLITONS}

We studied in the previous section the conditions under which input Gaussian spatiotemporal wave packets turn into ultrashort discrete solitons. In this section we study in detail the characteristic features of these few-cycle spatiotemporal

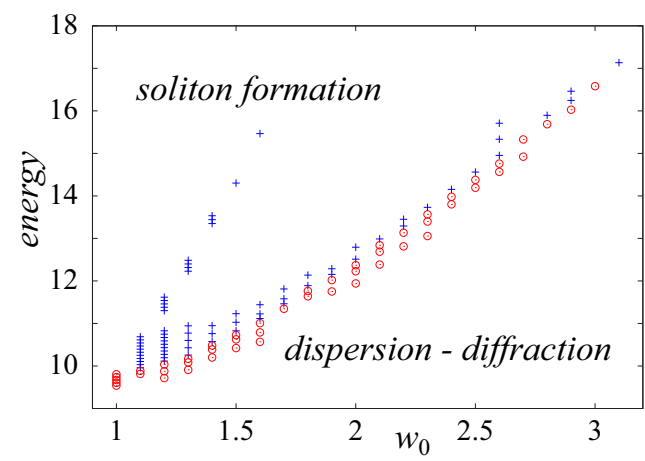

FIG. 5. Formation of solitons from a Gaussian input in the transverse width-energy plane, for $A_{0}=2.22$. Blue crosses: soliton forms; red circles: dispersion-diffraction occurs. 


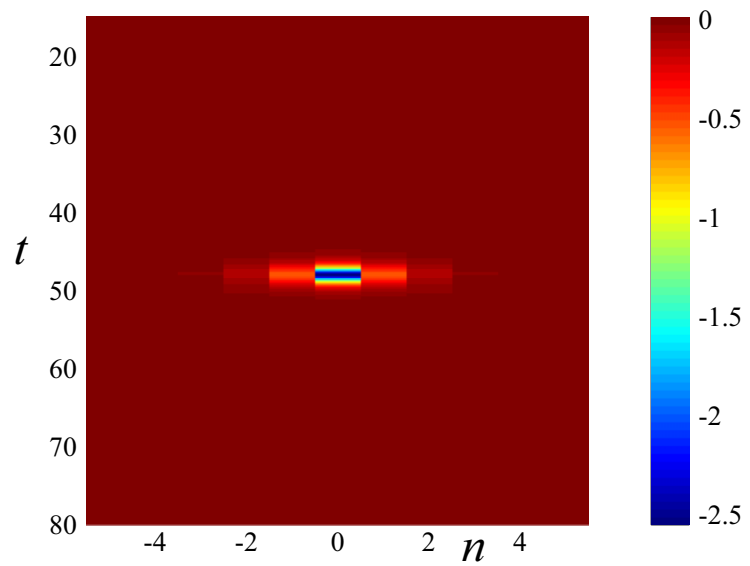

(a)

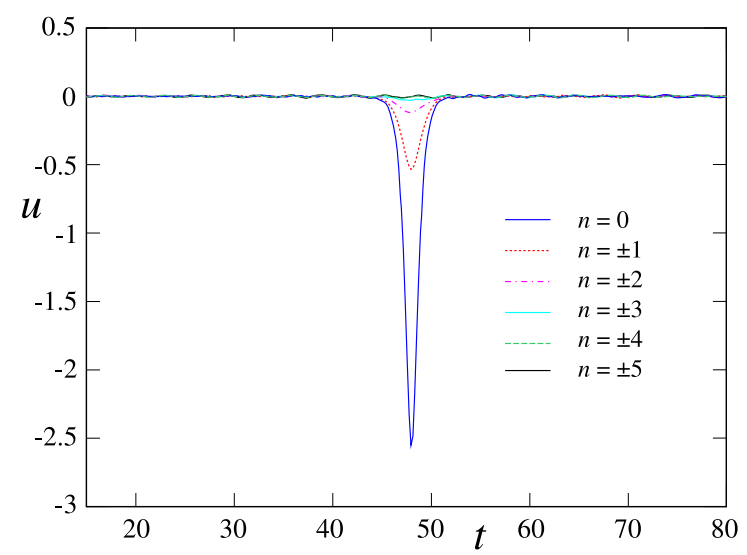

(b)

FIG. 6. Fundamental soliton with amplitude $\max _{t, n}\left(\left|u_{n}\right|\right)=$ 2.5667. (a) The optical field in the $(n, t)$ plane. (b) The temporal profile of the optical field.

optical solitons that form in waveguide arrays. The solitons are the stationary states of the nonlinear system (1); however, they are not constant, but oscillating, constituting the so-called breather solitons. Hence they cannot be computed directly by reducing the nonlinear coupled system of equations (1) to a set of ordinary differential equations. We run the propagation code until the soliton and the dispersive waves (or radiation) are well separated, then we replace the field at some distance from the soliton center with zero to remove the dispersive waves, and we repeat the operation until the amount of dispersive waves is low enough that the total energy of the optical field can be considered as being the energy of the emerging soliton, with an acceptable accuracy (in practice, we stop the procedure as the amplitude of the dispersive waves far from the pulse goes below $10^{-3}$ ).

We find that two kinds of discrete spatiotemporal solitons exist when computing them by using the above described numerical procedure: the breathing soliton, as expected when dealing with mKdV-type equations, but also the single-humped soliton, which is mainly the fundamental soliton of the $\mathrm{mKdV}$ equation, if we "forget" the transverse dimension.

The single-humped soliton is shown in Fig. 6. It is localized in both space and time and forms spontaneously from Gaussian spatiotemporal inputs after long enough propagation distance.

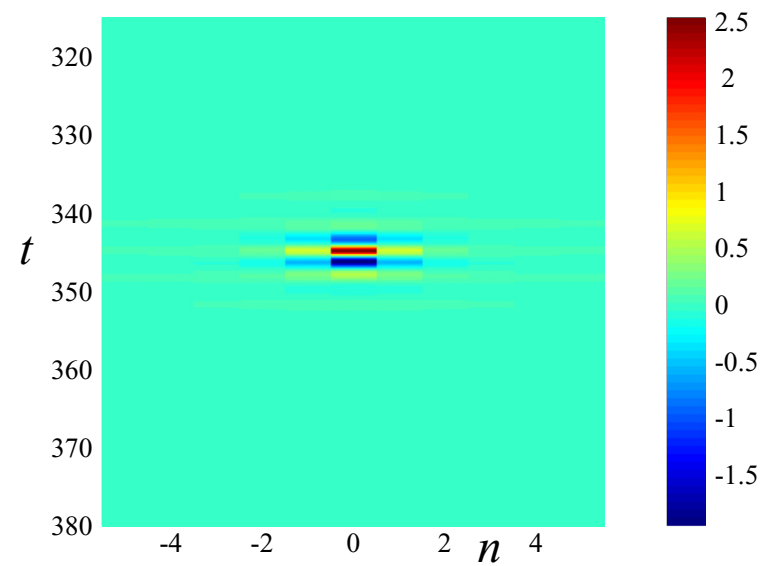

(a)

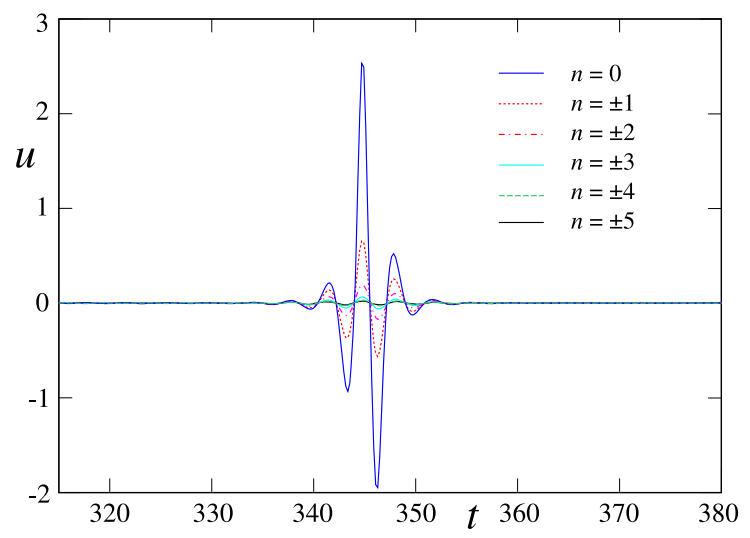

(b)

FIG. 7. Breathing soliton with amplitude $\max _{t, n}\left(\left|u_{n}\right|\right)=3.1801$. (a) The optical field in the $(n, t)$ plane. (b) The temporal profile of the optical breather.

The single-humped solitons can be either positive or negative, depending on the value of the initial phase. The breathing soliton is shown in Fig. 7; it is also localized in space and time, but it is an oscillating wave packet. The main characteristics of solitons, that is, their amplitude, energy, and duration, are recorded for a large number of numerical trials. The energy $E$ is plotted against the maximum amplitude $\max _{t, n}\left(\left|u_{n}\right|\right)$ in Fig. 8. It is seen from Fig. 8 that the energy of breathers is higher than the energy of fundamental solitons for the same maximum amplitude.

The soliton duration is computed using the standard deviation $\sigma=\sqrt{\left\langle t^{2}\right\rangle-\langle t\rangle^{2}}$, where the mean value $\langle\cdot\rangle$ is defined by

$$
\langle f(t)\rangle=\frac{\int_{-\infty}^{\infty} f(t) u^{4} d t}{\int_{-\infty}^{\infty} u^{4} d t} .
$$

(The usual definition, which involves a power 2 instead of 4 , leads to erroneous results, since it lends too much weight to the noisy background.) We consider only the central component $n=0$ that carries most of the energy, since the accuracy of the extraction of the pulse duration from numerical data strongly decreases with the signal-to-noise ratio. In order that the definition of soliton duration $\tau$ coincides with the half-width at $1 / e$ in the case where the pulse profile is of the hyperbolic 


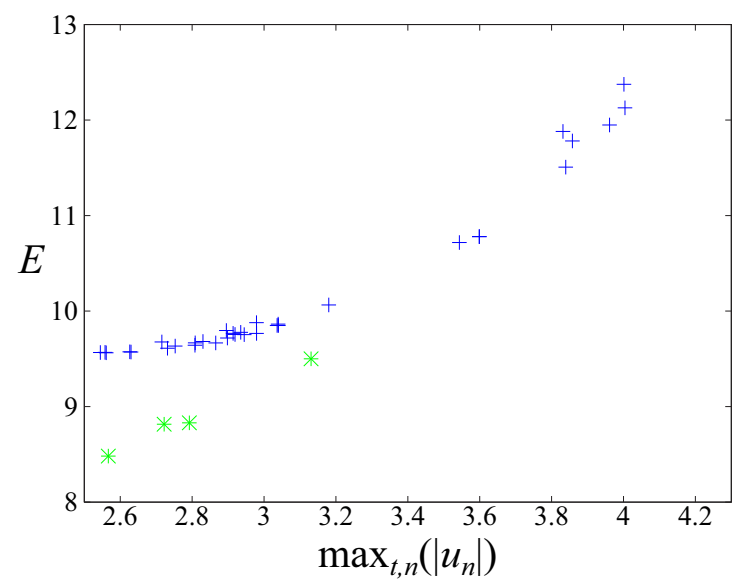

FIG. 8. The soliton energy $E$ as a function of the soliton's maximum amplitude $\max _{t, n}\left(\left|u_{n}\right|\right)$. Blue crosses: breathers; green stars: fundamental solitons.

secant type, we define it as

$$
\tau=2 \sigma \sqrt{\frac{3}{\pi^{2}-6}} .
$$

Then it is seen that the duration $\tau$ oscillates with $z$ due to the phase-carrier velocity mismatch. We denote by $\tau_{0}$ the average of $\tau$ over $z$. Comparison between the actual pulse profile and a hyperbolic secant with width $\tau$ or $\tau_{0}$ shows that only the mean value $\tau_{0}$ is relevant.

The duration $\tau_{0}$, which is computed this way, is shown in Fig. 9, versus the maximum amplitude $\max _{t, n}\left(\left|u_{n}\right|\right)$ of the solitons. It is seen that the duration slowly decreases as the maximum amplitude increases, and that the duration of the breathing solitons is about twice that of the single-humped ones. Since the duration decreases very slowly as the maximum amplitude increases, it is natural that the corresponding soliton energy then increases.

The spatial width of the soliton is rather small; most of its energy is concentrated in the central guide $n=0$. However, some finite amount of energy propagates in the lateral guides.

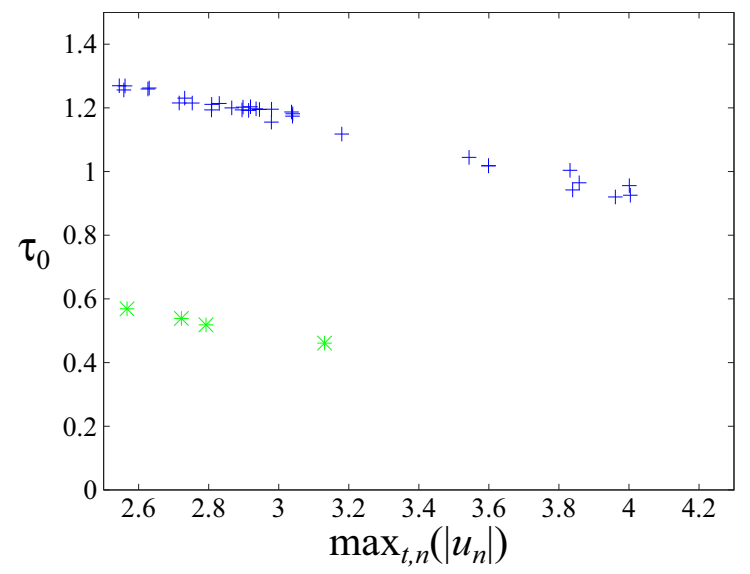

FIG. 9. The soliton duration $\tau_{0}$ as a function of the soliton's maximum amplitude $\max _{t, n}\left(\left|u_{n}\right|\right)$. Blue crosses: breathers; green stars: fundamental solitons.

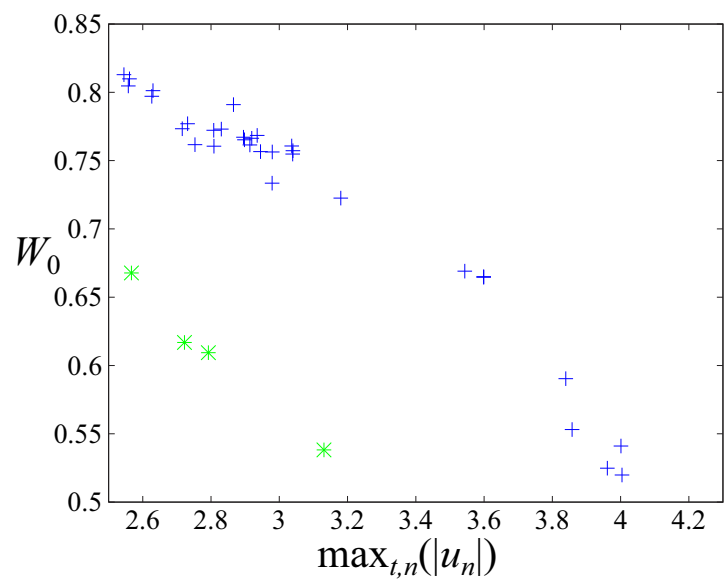

FIG. 10. The soliton spatial width $W_{0}$ as a function of the soliton's maximum amplitude $\max _{t, n}\left(\left|u_{n}\right|\right)$. Blue crosses: breathers; green stars: fundamental solitons.

This amount can be evaluated only by considering the amplitude in the few guides closest to the central ones (typically, $-2 \leqslant n \leqslant 2$, but in a few cases $-1 \leqslant n \leqslant 1$ only). Otherwise a reasonable accuracy cannot be reached, due to the presence of remaining dispersive waves. The transverse profile can be fitted by an expression of the form $\max _{t}\left|u_{n}\right|=A e^{-|n| / W_{0}}$, by applying a least-squares method to $\ln \left(\max _{t}\left|u_{n}\right|\right)$ for $0 \leqslant n \leqslant$ $2(0 \leqslant n \leqslant 1$ only for 4 among the 35 computed solitons). The results are shown in Fig. 10. It is seen that the solitons are indeed narrow, with $W_{0}<1$, and that the fundamental solitons are appreciably narrower than the breathers with the same maximum amplitude. We also observe from Fig. 10 that the width of the solitons decreases as their maximum amplitude grows; we note that the soliton duration also decreases slowly as the soliton maximum amplitude increases; see Fig. 9.

\section{CONCLUSION}

We have explored the existence and key features of ultrashort spatiotemporal optical solitons propagating in waveguide arrays, which are discrete solitons in the transverse direction, and few-cycle solitons in the longitudinal one. We have shown that such discrete-continuous few-cycle solitons can be of two types: either breathing solitons or single-humped ones. They form starting from an input Gaussian-type wave packet provided that the pulse energy is high enough. However, strictly speaking, an energy threshold does not exist. We also arrived at the conclusion that high energies of such discrete-continuous few-cycle solitons are reached for solitons with the smallest values of their duration; see Figs. 8 and 9. It was shown in [71] that nonlinear or dispersive coupling between waveguides, although they are negligible for long pulses, may have some appreciable importance in the fewcycle regime. An interesting problem for future research is to investigate how such coupling effects modify the formation and the structure of few-cycle pulse solitons in waveguide arrays. 
[1] D. N. Christodoulides, F. Lederer, and Y. Silberberg, Discretizing light behaviour in linear and nonlinear waveguide lattices, Nature (London) 424, 817 (2003).

[2] F. Lederer, G. I. Stegeman, D. N. Christodoulides, G. Assanto, M. Segev, and Y. Silberberg, Discrete solitons in optics, Phys. Rep. 463, 1 (2008).

[3] A. Szameit and S. Nolte, Discrete optics in femtosecondlaser-written photonic structures, J. Phys. B 43, 163001 (2010).

[4] D. Hennig and G. P. Tsironis, Wave transmission in nonlinear lattices, Phys. Rep. 307, 333 (1999).

[5] Y. V. Kartashov, B. A. Malomed, and L. Torner, Solitons in nonlinear lattices, Rev. Mod. Phys. 83, 247 (2011).

[6] P. G. Kevrekidis, K. O. Rasmussen, and A. R. Bishop, The discrete nonlinear Schrödinger equation: A survey of recent results, Int. J. Mod. Phys. B 15, 2833 (2001).

[7] P. G. Kevrekidis, The Discrete Nonlinear Schrödinger Equation: Mathematical Analysis, Numerical Computations and Physical Perspectives, Springer Tracts in Modern Physics Vol. 232 (Springer, Berlin, 2009).

[8] M. J. Ablowitz and J. F. Ladik, Nonlinear differential-difference equations, J. Math. Phys. 16, 598 (1975).

[9] A. Ankiewicz, N. Akhmediev and F. Lederer, Approach to firstorder exact solutions of the Ablowitz-Ladik equation, Phys. Rev. E 83, 056602 (2011).

[10] D. N. Christodoulides and R. I. Joseph, Discrete self-focusing in nonlinear arrays of coupled waveguides, Opt. Lett. 13, 794 (1988).

[11] H. S. Eisenberg, Y. Silberberg, R. Morandotti, A. R. Boyd, and J. S. Aitchison, Discrete Spatial Optical Solitons in Waveguide Arrays, Phys. Rev. Lett. 81, 3383 (1998).

[12] Y. S. Kivshar, Self-localization in arrays of defocusing waveguides, Opt. Lett. 18, 1147 (1993).

[13] A. B. Aceves, A. M. Rubenchik, S. K. Turitsyn, and C. De Angelis, Multidimensional solitons in fiber arrays, Opt. Lett. 19, 329 (1994).

[14] E. W. Laedke, K. H. Spatschek, and S. K. Turitsyn, Stability of Discrete Solitons and Quasicollapse to Intrinsically Localized Modes, Phys. Rev. Lett. 73, 1055 (1994).

[15] A. B. Aceves, G. G. Luther, C. De Angelis, A. M. Rubenchik, and S. K. Turitsyn, Energy Localization in Nonlinear Fiber Arrays: Collapse-Effect Compressor, Phys. Rev. Lett. 75, 73 (1995).

[16] A. B. Aceves, C. De Angelis, T. Peschel, R. Muschall, F. Lederer, S. Trillo, and S. Wabnitz, Discrete self-trapping, soliton interactions, and beam steering in nonlinear waveguide arrays, Phys. Rev. E 53, 1172 (1996).

[17] Z. Y. Xu, Y. V. Kartashov, L. C. Crasovan, D. Mihalache, and L. Torner, Spatiotemporal discrete multicolor solitons, Phys. Rev. E 70, 066618 (2004).

[18] D. Mihalache, D. Mazilu, F. Lederer, Y. V. Kartashov, L. C. Crasovan, and L. Torner, Stable three-dimensional spatiotemporal solitons in a two-dimensional photonic lattice, Phys. Rev. E 70, 055603 (2004).

[19] K. G. Makris, S. Suntsov, D. N. Christodoulides, G. I. Stegeman, and A. Haché, Discrete surface solitons, Opt. Lett. 30, 2466 (2005).

[20] N. C. Panoiu, R. M. Osgood, and B. A. Malomed, Semidiscrete composite solitons in arrays of quadratically nonlinear waveguides, Opt. Lett. 31, 1097 (2006).
[21] D. Mihalache, D. Mazilu, F. Lederer, and Y. Kivshar, Stable discrete surface light bullets, Opt. Express 15, 589 (2007).

[22] A. B. Aceves, O. V. Shtyrina, A. M. Rubenchik, M. P. Fedoruk, and S. K. Turitsyn, Spatiotemporal optical bullets in twodimensional fiber arrays and their stability, Phys. Rev. A 91, 033810 (2015).

[23] N. K. Efremidis and D. N. Christodoulides, Discrete GinzburgLandau solitons, Phys. Rev. E 67, 026606 (2003).

[24] N. K. Efremidis and D. N. Christodoulides, and K. Hizanidis, Two-dimensional discrete Ginzburg-Landau solitons, Phys. Rev. A 76, 043839 (2007).

[25] J. M. Soto-Crespo, N. Akhmediev, and A. Ankiewicz, Motion and stability properties of solitons in discrete dissipative structures, Phys. Lett. A 314, 126 (2003).

[26] D. Mihalache, D. Mazilu, and F. Lederer, Spatiotemporal discrete Ginzburg-Landau solitons in two-dimensional photonic lattices, Eur. Phys. J. Spec. Top. 173, 255 (2009).

[27] B. A. Malomed and P. G. Kevrekidis, Discrete vortex solitons, Phys. Rev. E 64, 026601 (2001).

[28] H. Leblond, B. A. Malomed, and D. Mihalache, Spatiotemporal vortices in optical fiber bundles, Phys. Rev. A 77, 063804 (2008).

[29] H. Leblond, B. A. Malomed, and D. Mihalache, Spatiotemporal vortex solitons in hexagonal arrays of waveguides, Phys. Rev. A 83, 063825 (2011).

[30] J. W. Fleischer, M. Segev, N. K. Efremidis, and D. N. Christodoulides, Observation of two-dimensional discrete solitons in optically induced nonlinear photonic lattices, Nature (London) 422, 147 (2003).

[31] D. Neshev, E. Ostrovskaya, Yu. Kivshar, and W. Krolikowski, Spatial solitons in optically induced gratings, Opt. Lett. 28, 710 (2003).

[32] D. N. Neshev, T. J. Alexander, E. A. Ostrovskaya, and Yu. S. Kivshar, Observation of Discrete Vortex Solitons in Optically Induced Photonic Lattices, Phys. Rev. Lett. 92, 123903 (2004).

[33] S. Suntsov, K. G. Makris, D. N. Christodoulides, G. I. Stegeman, H. Haché, R. Morandotti, H. Yang, G. Salamo, and M. Sorel, Observation of Discrete Surface Solitons, Phys. Rev. Lett. 96, 063901 (2006).

[34] A. Szameit, Y. V. Kartashov, F. Dreisow, T. Pertsch, S. Nolte, A. Tünnermann, and L. Torner, Observation of Two-Dimensional Surface Solitons in Asymmetric Waveguide Arrays, Phys. Rev. Lett. 98, 173903 (2007).

[35] M. Heinrich, A. Szameit, F. Dreisov, R. Keil, S. Minardi, T. Pertsch, S. Nolte, A. Tünnermann, and F. Lederer, Observation of Three-Dimensional Discrete-Continuous X Waves in Photonic Lattices, Phys. Rev. Lett. 103, 113903 (2009).

[36] S. Minardi, F. Eilenberger, Y. V. Kartashov, A. Szameit, U. Röpke, J. Kobelke, K. Schuster, H. Bartelt, S. Nolte, L. Torner, F. Lederer, A. Tünnermann, and T. Pertsch, Three-Dimensional Light Bullets in Arrays of Waveguides, Phys. Rev. Lett. 105, 263901 (2010).

[37] F. Eilenberger, K. Prater, S. Minardi, R. Geiss, U. Röpke, J. Kobelke, K. Schuster, H. Bartelt, S. Nolte, A. Tünnermann, and T. Pertsch, Observation of Discrete, Vortex Light Bullets, Phys. Rev. X 3, 041031 (2013).

[38] T. Brabec and F. Krausz, Nonlinear Optical Pulse Propagation in the Single-Cycle Regime, Phys. Rev. Lett. 78, 3282 (1997).

[39] S. Amiranashvili, U. Bandelow, and N. Akhmediev, Dispersion of nonlinear group velocity determines shortest envelope solitons, Phys. Rev. A 84, 043834 (2011). 
[40] A. V. Husakou and J. Herrmann, Supercontinuum Generation of Higher-Order Solitons by Fission in Photonic Crystal Fibers, Phys. Rev. Lett. 87, 203901 (2001).

[41] S. Amiranashvili, U. Bandelow, and N. Akhmediev, Few-cycle optical solitary waves in nonlinear dispersive media, Phys. Rev. A 87, 013805 (2013).

[42] E. V. Kazantseva, A. I. Maimistov, and J.-G. Caputo, Reduced Maxwell-Duffing description of extremely short pulses in nonresonant media, Phys. Rev. E 71, 056622 (2005).

[43] N. N. Rosanov, V. V. Kozlov, and S. Wabnitz, Maxwell-DrudeBloch dissipative few-cycle optical solitons, Phys. Rev. A 81, 043815 (2010).

[44] S. V. Sazonov, On the nonlinear optics of few-cycle pulses, Bull. Russ. Acad. Sci. 75, 157 (2011).

[45] H. Leblond, H. Triki, and D. Mihalache, Theoretical studies of ultrashort-soliton propagation in nonlinear optical media from a general quantum model, Rom. Rep. Phys. 65, 925 (2013).

[46] H. Leblond and D. Mihalache, Models of few optical cycle solitons beyond the slowly varying envelope approximation, Phys. Rep. 523, 61 (2013).

[47] D. J. Frantzeskakis, H. Leblond, and D. Mihalache, Nonlinear optics of intense few-cycle pulses: An overview of recent theoretical and experimental developments, Rom. J. Phys. 59, 767 (2014).

[48] I. V. Mel'nikov, D. Mihalache, F. Moldoveanu, and N.-C. Panoiu, Quasiadiabatic following of femtosecond optical pulses in a weakly excited semiconductor, Phys. Rev. A 56, 1569 (1997).

[49] T. Schäfer and C. E. Wayne, Propagation of ultra-short optical pulses in cubic nonlinear media, Physica D: Nonlinear Phenom. 196, 90 (2004).

[50] A. Sakovich and S. Sakovich, The short pulse equation is integrable, J. Phys. Soc. Jpn. 74, 239 (2005).

[51] J. C. Brunelli, The short pulse hierarchy, J. Math. Phys. 46, 123507 (2005).

[52] H. Leblond and F. Sanchez, Models for optical solitons in the two-cycle regime, Phys. Rev. A 67, 013804 (2003).

[53] A. Nazarkin, Nonlinear Optics of Intense Attosecond Light Pulses, Phys. Rev. Lett. 97, 163904 (2006).

[54] H. Leblond, H. Triki, and D. Mihalache, Derivation of a generalized double sine-Gordon equation describing ultrashort soliton propagation in optical media composed of multilevel atoms, Phys. Rev. A 86, 063825 (2012).

[55] H. Leblond, S. V. Sazonov, I. V. Mel'nikov, D. Mihalache, and F. Sanchez, Few-cycle nonlinear optics of multicomponent media, Phys. Rev. A 74, 063815 (2006).

[56] H. Leblond and D. Mihalache, Few-optical-cycle solitons: Modified Korteweg-de Vries sine-Gordon equation versus other non-slowly-varying-envelope-approximation models, Phys. Rev. A 79, 063835 (2009).

[57] S. P. Popov, Numerical analysis of soliton solutions of the modified Korteweg-de Vries-sine-Gordon equation, Comput. Math. Phys. 55, 437 (2015).
[58] S. V. Sazonov, Extremely short and quasi-monochromatic electromagnetic solitons in a two-component medium, J. Exp. Theor. Phys. 92, 361 (2001).

[59] V. G. Bespalov, S. A. Kozlov, Yu. A. Shpolyanskiy, and I. A. Walmsley, Simplified field wave equations for the nonlinear propagation of extremely short light pulses, Phys. Rev. A 66, 013811 (2002).

[60] S. A. Skobelev, D. V. Kartashov, and A. V. Kim, Few-OpticalCycle Solitons and Pulse Self-Compression in a Kerr Medium, Phys. Rev. Lett. 99, 203902 (2007).

[61] S. V. Sazonov and N. V. Ustinov, Extremely short vector solitons under the conditions of conical refraction, JETP Lett. 99, 503 (2014).

[62] S. Amiranashvili, U. Bandelow, and N. Akhmediev, Ultrashort optical solitons in transparent nonlinear media with arbitrary dispersion, Opt. Quantum Electron. 46, 1233 (2014).

[63] H. Leblond, D. Kremer, and D. Mihalache, Ultrashort spatiotemporal optical solitons in quadratic nonlinear media: Generation of line and lump solitons from few-cycle input pulses, Phys. Rev. A 80, 053812 (2009).

[64] B. A. Malomed, D. Mihalache, F. Wise, and L. Torner, Spatiotemporal optical solitons, J. Opt. B 7, R53 (2005).

[65] D. Mihalache, Linear and nonlinear light bullets: Recent theoretical and experimental studies, Rom. J. Phys. 57, 352 (2012).

[66] D. Mihalache, Multidimensional localized structures in optics and Bose-Einstein condensates: A selection of recent studies, Rom. J. Phys. 59, 295 (2014).

[67] D. Mihalache, Localized structures in nonlinear optical media: A selection of recent studies, Rom. Rep. Phys. 67, 1383 (2015).

[68] B. Malomed, L. Torner, F. Wise, and D. Mihalache, On multidimensional solitons and their legacy in contemporary atomic, molecular and optical physics, J. Phys. B 49, 170502 (2016).

[69] B. A. Malomed, Multidimensional solitons: Well-established results and novel findings, Eur. Phys. J. Special Topics 225, 2507 (2016).

[70] D. Mihalache, Multidimensional localized structures in optical and matter-wave media: A topical survey of recent literature, Rom. Rep. Phys. 69, 403 (2017).

[71] H. Leblond and S. Terniche, Waveguide coupling in the fewcycle regime, Phys. Rev. A 93, 043839 (2016).

[72] S. Terniche, H. Leblond, D. Mihalache, and A. Kellou, Fewcycle optical solitons in linearly coupled waveguides, Phys. Rev. A 94, 063836 (2016).

[73] D. N. Christodoulides, I. Ch. Khoo, G. J. Salamo, G. I. Stegeman, and E. W. Van Stryland, Nonlinear refraction and absorption: mechanisms and magnitudes, Adv. Opt. Photon. 2, 60 (2010).

[74] G. Boudebs, S. Cherukulappurath, M. Guignard, J. Troles, F. Smektala, and F. Sanchez, Linear optical characterization of chalcogenide glasses, Opt. Commun. 230, 331 (2004).

[75] C. Quémard, F. Smektala, V. Couderc, A. Barthélémy, and J. Lucas, Chalcogenide glasses with high nonlinear optical properties for telecommunications, J. Phys. Chem. Solids 62, 1435 (2001). 